\title{
A Binational Influenza Surveillance Network - California/Baja California
}

\author{
Esmeralda Iniguez-Stevens*, Sarah Marikos and Karen Ferran \\ EWIDS, California Department of Public Health, San Diego, CA, USA
}

\section{Objective}

To enhance cross-border surveillance for Influenza-Like-Illness (ILI) in the California/Baja California $(\mathrm{CA} / \mathrm{BC})$ border region through the formation of a border binational surveillance network.

\section{Introduction}

In response to the $2009 \mathrm{H} 1 \mathrm{~N} 1$ pandemic, the Early Warning Infectious Disease Surveillance Program (EWIDS) Office of Binational Border Health in the California Department of Public Health sought to strengthen outpatient ILI surveillance along the $\mathrm{CA} / \mathrm{BC}$ border by creating the first binational influenza surveillance network in the region. The establishment of this network was crucial for enhancing cross-border situational awareness of influenza activity, especially in a region characterized by high levels of population mobility.

\section{Methods}

During summer of 2009, an assessment of current ILI surveillance activities in the $\mathrm{CA} / \mathrm{BC}$ border region was conducted. Findings were utilized to guide recruitment efforts and build a cross-border surveillance network. In CA the assessment revealed that sentinel sites in the border region participating in CDC's ILINet surveillance program were primarily pediatric or school-based clinics and that family practice patients were not equally represented. In BC the need to enhance surveillance among the private sector was identified, particularly among patients belonging to binational healthcare service plans. These plans offer care to US workforce individuals who seek medical care in BC. Other needs identified included the need to enhance surveillance among underserved populations such as farm workers and tribal communities which were not currently being represented. Working together with partners from both sides of the border EWIDS initiated efforts to address identified gaps. Over a three-year period EWIDS recruited private and public sector clinics to participate in the network.

\section{Results}

As a result of the assessment recruitment efforts were focused on inviting family practice clinics, private clinics, tribal health centers and clinics that provide care to underserved populations to participate in the network. These efforts led to the establishment of the California/Baja California Border Outpatient Provider ILI Surveillance Network, which monitors syndromic and virologic influenza activity. In total EWIDS recruited 22 (13 in CA, 9 in BC) sentinel sites to participate; of these, 17 are family practice sites and 5 are pediatric sites. Additionally, prior to the EWIDS enhancement local tribal health clinics were not represented in the surveillance system. EWIDS efforts resulted in the inclusion of 8 tribal sites in CA and 1 in BC. Figure 1 shows the geographical location of network sites, which includes sites recruited by EWIDS post-assessment as well as pre- existing sites. Over the past three influenza seasons (2009-2012) EWIDS recruited sites have constituted $47 \%$ of all network sites. Since the 2009-2010 influenza season 483,772 individuals have been screened for ILI by participating sites; of these, $65.8 \%(n=318,295)$ were screened by EWIDS recruited sites. Since the establishment of the network EWIDS has focused on sentinel site retention, logistical support, data collection, and dissemination of surveillance results. A weekly report summarizing syndromic and virologic activity is distributed to public health officials throughout the influenza season.

\section{Conclusions}

The network serves as an example of a successful binational coordinated effort to establish an early warning system for enhancing situational awareness of influenza activity in a cross-border setting. Next steps include conducting a formal evaluation of the existing surveillance system, enhancing specimen collection for virologic testing, and continuing to foster and build public/private partnerships.

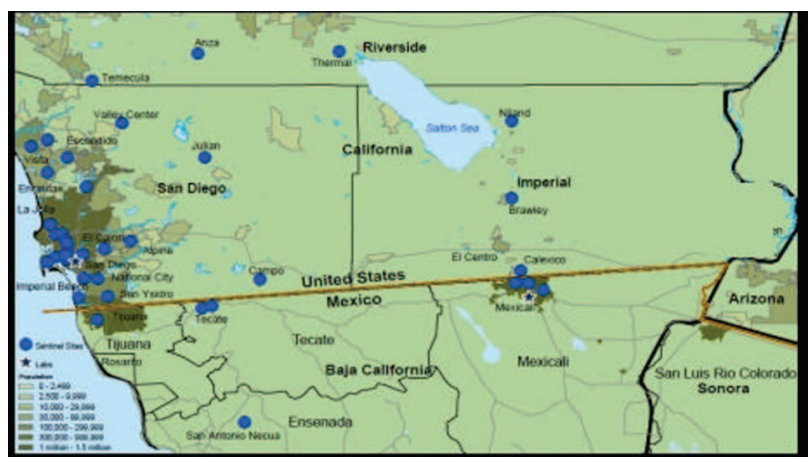

Figure 1. Surveillance Network

\section{Keywords}

influenza; surveillance; syndromic; virologic; binational

\section{Acknowledgments}

We gratefully acknowledge all our border partners for their contributions and support including: Border Infectious Disease Surveillance Program, Imperial County Public Health Department, County of San Diego Public Health Services Epidemiology \& Immunization Services Branch, Naval Health Research Center Laboratory, California Department of Public Health Center for Infectious Diseases, Laboratorio Estatal de Salud Publica de Baja California, Departamento de Epidemiologia Estatal Baja California, and our medical partners.

\section{*Esmeralda Iniguez-Stevens}

E-mail: einiguez@cdph.ca.gov 